\title{
Luminescent and photovoltaic properties of poly(9,9-dioctylfluorene- co-bithiophene) in organic electronic devices
}

\author{
XU XiaoWei ${ }^{1}$, ZHU EnWei ${ }^{2}$, BIAN LinYi ${ }^{2}$, WANG ZiXuan ${ }^{1}$, WANG Jin ${ }^{1}$, ZHUO ZuLiang ${ }^{1}$, \\ WANG Jian $^{1}$, ZHANG FuJun $^{1 *} \&$ TANG WeiHua ${ }^{2 *}$ \\ ${ }^{1}$ Key Laboratory of Luminescence and Optical Information, Ministry of Education, Beijing Jiaotong University, Beijing 100044, China; \\ ${ }^{2}$ Key Laboratory for Soft Chemistry and Functional Materials, Ministry of Education, Nanjing University of Science and Technology, Nanjing \\ 210094, China
}

Received August 19, 2011; accepted October 21, 2011

\begin{abstract}
We studied the luminescent and photovoltaic properties of poly(9,9-dioctylfluorene-co-bithiophene) (F8T2) based on ITO/ PEDOT:PSS/F8T2/Bphen/LiF(0 or $1 \mathrm{~nm}$ )/Al and ITO/PEDOT:PSS/F8T2:PCBM/Bphen/Al. A stable and bright yellow emission was obtained from polymer F8T2, and the electroluminescence power reached $45 \mu \mathrm{W}$ at a $15 \mathrm{~V}$ driving voltage. Polymer F8T2 shows a broad absorption band from 400 to $500 \mathrm{~nm}$, and has a shorter absorption edge at about $560 \mathrm{~nm}$ compared to that of the typical electron donor P3HT $(650 \mathrm{~nm})$. The photoluminescence quenching of F8T2 occurs with only a small fraction of blended PCBM due to the effective exciton dissociation at the interface between F8T2 and PCBM. Polymer solar cells (PSCs) using F8T2: PCBM as the active layer show a low power conversion efficiency (PCE) of $0.10 \%$ with an open circuit voltage ( $V_{\text {oc }}$ ) of $0.91 \mathrm{~V}$ and short circuit current density $\left(J_{\mathrm{sc}}\right)$ of $0.23 \mathrm{~mA} / \mathrm{cm}^{2}$. The PSCs using F8T2:P3HT:PCBM as the active layer have a $V_{\text {oc }}$ of $0.85 \mathrm{~V}$ and $J_{\text {sc }}$ of $3.02 \mathrm{~mA} / \mathrm{cm}^{2}$, improving the PCE by about $0.90 \%$. We attribute the improved cell performance to the higher number of photons harvested by P3HT molecules.
\end{abstract}

energy level alignment, polymer light emitting diodes, polymer solar cells

Citation: $\quad \mathrm{Xu} \mathrm{X} \mathrm{W,} \mathrm{Zhu} \mathrm{E} \mathrm{W,} \mathrm{Bian} \mathrm{L} \mathrm{Y,} \mathrm{et} \mathrm{al.} \mathrm{Luminescent} \mathrm{and} \mathrm{photovoltaic} \mathrm{properties} \mathrm{of} \mathrm{poly(9,9-dioctylfluorene-co-bithiophene)} \mathrm{in} \mathrm{organic} \mathrm{electronic} \mathrm{devices.}$ Chin Sci Bull, 2012, 57: 970-975, doi: 10.1007/s11434-011-4964-3

Organic optoelectronic devices have developed rapidly during the past few decades because of their advantages of easy large-area fabrication, light weight, and low fabrication cost. The operational model of organic light emitting diodes (OLEDs) is reverse with polymer solar cells (PSCs). The aim of research on both kinds of devices is high conversion efficiency from electricity to light or the reverse. Recently, the power conversion efficiencies (PCEs) of PSCs has reached $7 \%$ using narrow bandgap poly[4,8-bis-substitutedbenzo[1,2-b:4,5-b']dithiophene-2,6-diyl-alt-4-substitutedthieno [3,4-b] thiophene-2,6-diyl] (PBDTTT)-derived polymers doped with $(6,6)$-phenyl $\mathrm{C}_{60}$ butyric acid methyl ester (PCBM) under the standard solar spectrum AM1.5G [1,2].

*Corresponding authors (email: fjzhang@bjtu.edu.cn; whtang@mail.njust.edu.cn)
The electron donor band gap and energy level alignment between donor and acceptor strongly influence the absorption, exciton dissociation and charge transfer, finally determining the PCE of PSCs [3-5]. Fullerene and its derivatives are considered to be the best electron acceptors so far, which is attributed to its ultrafast photo-induced charge transfer, high electron mobility, and better phase separation in the blend films [6,7]. The lowest unoccupied molecular orbit (LUMO) of typical donor material P3HT is about $0.5 \mathrm{eV}$ higher than that of PCBM, which favors electron transfer from P3HT to PCBM. However, the relatively high-lying highest occupied molecular orbit (HOMO) limits the maximum open-circuit voltage $\left(V_{\mathrm{oc}}\right)$ and causes oxidation instability of the cells at ambient conditions [4]. To further improve the photovoltaic propriety of PSCs, effort should be 
devoted to the precise control of the HOMO and LUMO energy levels, structural modification for forming a preferred morphology in the blend film with PCBM, strong and broad visible absorption with high charge carrier mobility, and structural regularity [8]. The HOMO and LUMO energy levels of F8T2 are $-5.5 \mathrm{eV}$ and $-3.1 \mathrm{eV}$, which shows a relatively larger band gap of $2.4 \mathrm{eV}$. The energy barrier between the HOMO of F8T2 and LUMO of PCBM is about $1.8 \mathrm{eV}$, which favors obtaining a higher $V_{\text {oc }}$ from F8T2: PCBM-based cells. The energy difference between the LUMOs of the donor and acceptor should be larger than $0.3 \mathrm{eV}$ for efficient charge separation, which strongly determines the short circuit current density $\left(J_{\mathrm{sc}}\right)$ of cells $[9,10]$. The charge separation should be effective according to the LUMO energy levels of F8T2 and PCBM. Based on the above information on F8T2, we fabricated PSCs with F8T2: PCBM or F8T2:P3HT:PCBM as the active layers. It is worth noting that F8T2's properties are very similar to those of poly[2-methoxy-5-(2'-ethylhexyloxy)-1,4-phenylenevinylene] (MEH-PPV), a workhorse material commonly used in PSCs and OLEDs $[11,12]$. PSCs using MEH-PPV as the donor show a bigger $V_{\mathrm{oc}}$ and limited $J_{\mathrm{sc}}$ compared to PSCs with P3HT as the donor material $[13,14]$. A series of interfacial layers, $\mathrm{LiF}, \mathrm{MoO}_{3}$ and $\mathrm{ZnO}$, were applied to improve device performances [15-19]. In this study, we used ultrathin $\mathrm{LiF}$ interfacial layer to improve OLED performance and Bphen interfacial layer to enhance PSC performance.

We studied the luminescent and photovoltaic performance of F8T2 using ITO/PEDOT:PSS/F8T2/Bphen/LiF (0 or $1 \mathrm{~nm}) / \mathrm{Al}$ and ITO/PEDOT:PSS/F8T2: PCBM (F8T2: P3HT:PCBM)/Bphen/Al. Experimental results for F8T2 show a bright yellow emission under different driving voltages. We obtained the photovoltaic performance of F8T2 by doping with PCBM. To optimize the PSC's performance, the typical electron donor P3HT was doped with F8T2: PCBM to improve the photon harvesting. We studied the effect of different thicknesses of a Bphen interfacial layer on PSC performance. After the interfacial engineering on the OLEDs and PSCs, the maximum PCE of PSCs reached $0.90 \%$ under illumination at $100 \mathrm{~mW} / \mathrm{cm}^{2}$, and OLED intensity reached $45 \mu \mathrm{W}$ at a $15 \mathrm{~V}$ driving voltage.

\section{Experimental}

The ITO substrates (sheet resistance $15 \Omega / \square$ ) were cleaned consecutively in ultrasonic baths containing acetone, ethanol and de-ionized water and dried by high speed nitrogen gas. The cleaned substrates were treated by UV light ozone for $10 \mathrm{~min}$ to improve the ITO work function. A thin layer of poly-(3,4-ethylenedioxythiophene)-poly-(styrenesulfonate) (PEDOT:PSS) was spin-coated on the substrates at a speed of $3000 \mathrm{r} / \mathrm{min}$ for $40 \mathrm{~s}$. The ITO substrates coated with
PEDOT:PSS were transferred to a hot plate and annealed at $120^{\circ} \mathrm{C}$ for $10 \mathrm{~min}$. The polymer materials F8T2, P3HT and PCBM were dissolved in chloroform with a concentration $10 \mathrm{mg} / \mathrm{mL}$. Their blend solution was spin-coated on PEDOT: PSS-coated ITO substrates as the active layer. The pristine F8T2 solution was spin-coated on PEDOT:PSS-coated ITO substrates and used as the active layer for OLEDs. Subsequently, the substrates coated with different active layers were transferred to a vacuum chamber, and an interfacial layer of 4,7-diphenyl-1,10-phenanthroline (Bphen) was deposited on the active layer under $5 \times 10^{-4} \mathrm{~Pa}$. The LiF ultrathin film $(1 \mathrm{~nm})$ was deposited on the Bphen layer under $5 \times 10^{-3} \mathrm{~Pa}$. The rate of deposition and final film thickness were monitored by a quartz crystal microbalance. The thickness $100 \mathrm{~nm}$ aluminum cathode was evaporated under $5 \times$ $10^{-3} \mathrm{~Pa}$.

The absorption spectra of all films were measured by a Shimadzu UV-3101 PC spectrometer. The photoluminescence (PL) spectra were measured by a PerkinElmer LS55 fluorescence spectrometer. The current-voltage $(I-V)$ characteristics of PSCs were measured under illumination of $100 \mathrm{~mW} / \mathrm{cm}^{2}$ using a $150 \mathrm{~W}$ xenon lamp. The luminescence power and $I-V$ characteristics of OLEDs were measured by a Keithley source meter 2410. The electroluminescence (EL) spectra were recorded by charge coupled device. Figure 1 shows the chemical structures of the materials, OLED schematic, and energy level alignment of the materials.

\section{Results and discussion}

\subsection{The luminescence properties of F8T2}

EL spectra were measured under different driving voltages and are shown in the Figure 2. To confine the charge carrier's recombination zone in the F8T2 layer, a hole blocking layer of Bphen with a higher HOMO level $(-6.1 \mathrm{eV})$ was inserted between F8T2 and LiF, as shown in Figure 1(e). The EL emission of F8T2 shows a bright orange emission peaking at $570 \mathrm{~nm}$ and a shoulder emission peaking at 605 $\mathrm{nm}$, which is identical with its PL spectra under excitation of $450 \mathrm{~nm}$ light. Figure 3 shows the current density and EL power versus driving voltage characteristics of two kinds of OLEDs with and without LiF ultrathin layer. It appears that the EL intensity of F8T2 was markedly improved by the insertion of the $1 \mathrm{~nm} \mathrm{LiF}$ layer, which reaches $45 \mu \mathrm{W}$ under $15 \mathrm{~V}$. The turn-on voltage of OLEDs with a $1 \mathrm{~nm} \mathrm{LiF}$ layer was decreased compared to the OLEDs without LiF layer, which could be attributed to the increase of electron tunneling into F8T2 layer due to the insertion of the $\mathrm{LiF}$ layer. The several potential functions of $\mathrm{LiF}$ ultrathin layer are already reported and are: (1) as a buffer, reducing the hot Al-bonding-induced degradation of the organic layer [7]; (2) dissociation of $\mathrm{LiF}$ resulting in doping of the organic layer and/or alloying of the metal cathode [20,21]; (3) forming a 

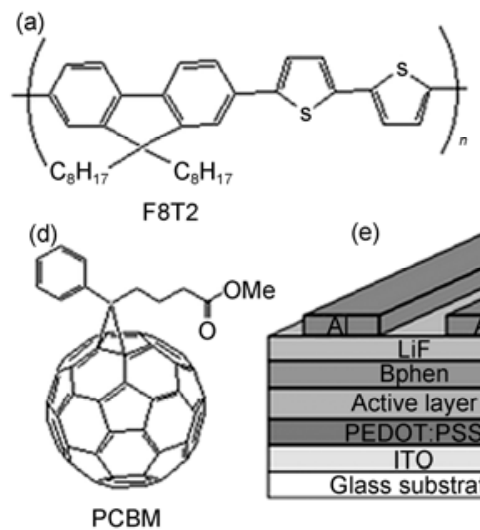

(e)<smiles>CCCCCCc1cc(C(C)(C)C)sc1-c1cc(CCCCCC)c(C(C)(C)C)s1</smiles>

P3HT

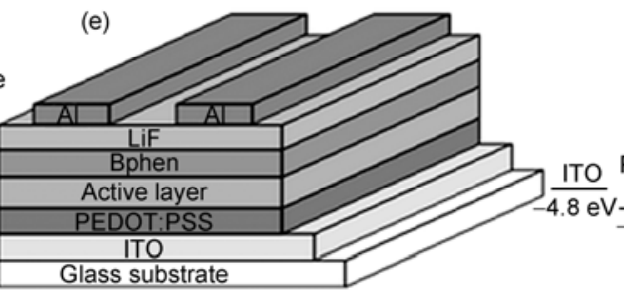

(f)
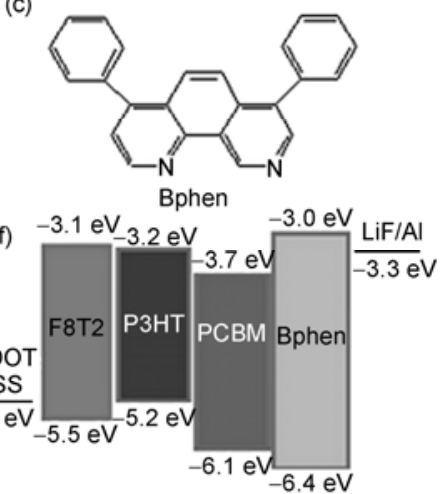

Figure 1 Organic materials used. (a) F8T2; (b) P3HT; (c) Bphen; (d) PCBM; (e) schematic of polymer light emitting diodes; (f) energy level alignment materials used.

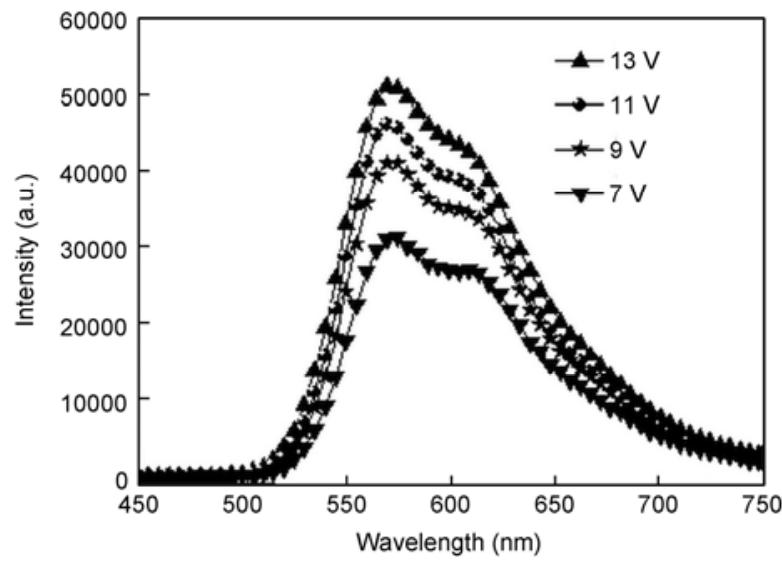

Figure 2 EL spectra of ITO/PEDOT:PSS/F8T2/Bphen/LiF/Al under different driving voltages.

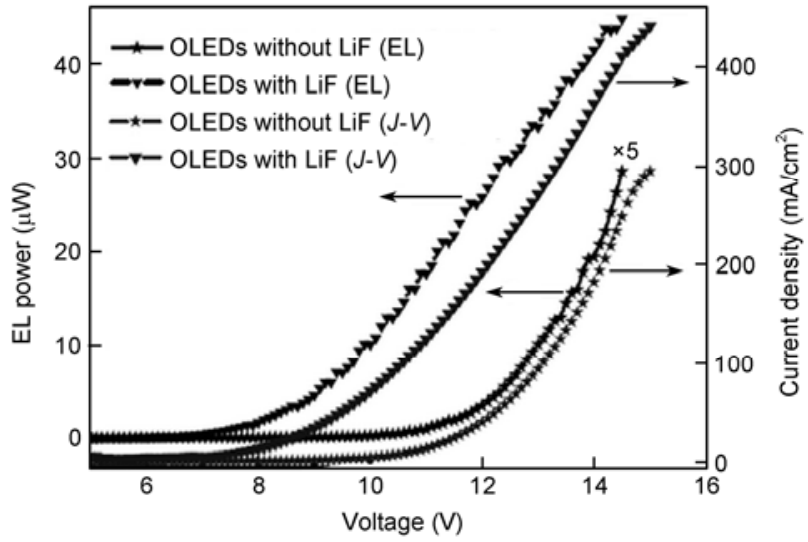

Figure 3 EL intensity and current density dependence on the driving voltage of ITO/PEDOT: PSS/F8T2/Bpehn/LiF(0 or $1 \mathrm{~nm}) / \mathrm{Al}$.

dipole layer decreasing the electron injection barriers [22]. Lu et al. investigated the chemical structure of the interface between the $\mathrm{Al} / \mathrm{LiF}$ bilayer cathode and tris(8-hydroxyquinoline) aluminum by using X-ray photoelectron spectroscopy
[22]. There are two kinds of $\mathrm{F}$ at the interface; one is attributed to $\mathrm{LiF}$ and the other $\mathrm{F}$ attached to the $\mathrm{Alq}_{3}$. This F-doped $\mathrm{Alq}_{3}$ layer could induce a downshift in molecular orbital levels and thus lead to a reduced electron injection barrier [23]. In this experiment, the hole current should remain constant because the same hole injection barriers are used for all OLEDs. The $\mathrm{LiF}$ layer could improve electron injection because of the lowered electron injection barrier, which could be supported by the increase in current density of OLEDs with LiF layers at the same driving voltage.

\subsection{Photovoltaic properties of F8T2}

Organic electronic materials have attracted much attention because of their versatile functions including bidirectional conversion between electric and light. The HOMO and LUMO energy levels of organic electronic materials are the main parameters determining the performance of organic electronic devices [24,25]. It is known that the $V_{\text {oc }}$ of PSCs is determined by either the difference between donor HOMO and acceptor LUMO or the work function difference of the electrodes or both $[7,14]$. The energy difference between the LUMOs of donor and acceptor should be larger than $0.3 \mathrm{eV}$ for efficient charge separation, which strongly determines the $J_{\text {sc }}$ of cells.

We studied the photovoltaic characteristics of F8T2 and F8T2:P3HT: by doping with the commonly used electron acceptor PCBM. The UV-visible absorption spectra of F8T2 and F8T2:P3HT doped with PCBM were measured and are shown in Figure 4(a). Polymer F8T2 shows a broader absorption range from 400 to $500 \mathrm{~nm}$, and has a shorter absorption edge at about $560 \mathrm{~nm}$ compared to that of the typical electron donor P3HT (650 nm) [26]. The blended polymer F8T2:P3HT thin films show much a broader absorption ranges from 400 to $650 \mathrm{~nm}$, which is attributed to the absorption spectra overlapping between F8T2 and P3HT. The PL spectra of F8T2, F8T2:P3HT, PCBM and their thin films blends were measured and are shown in Figure 4(b). 

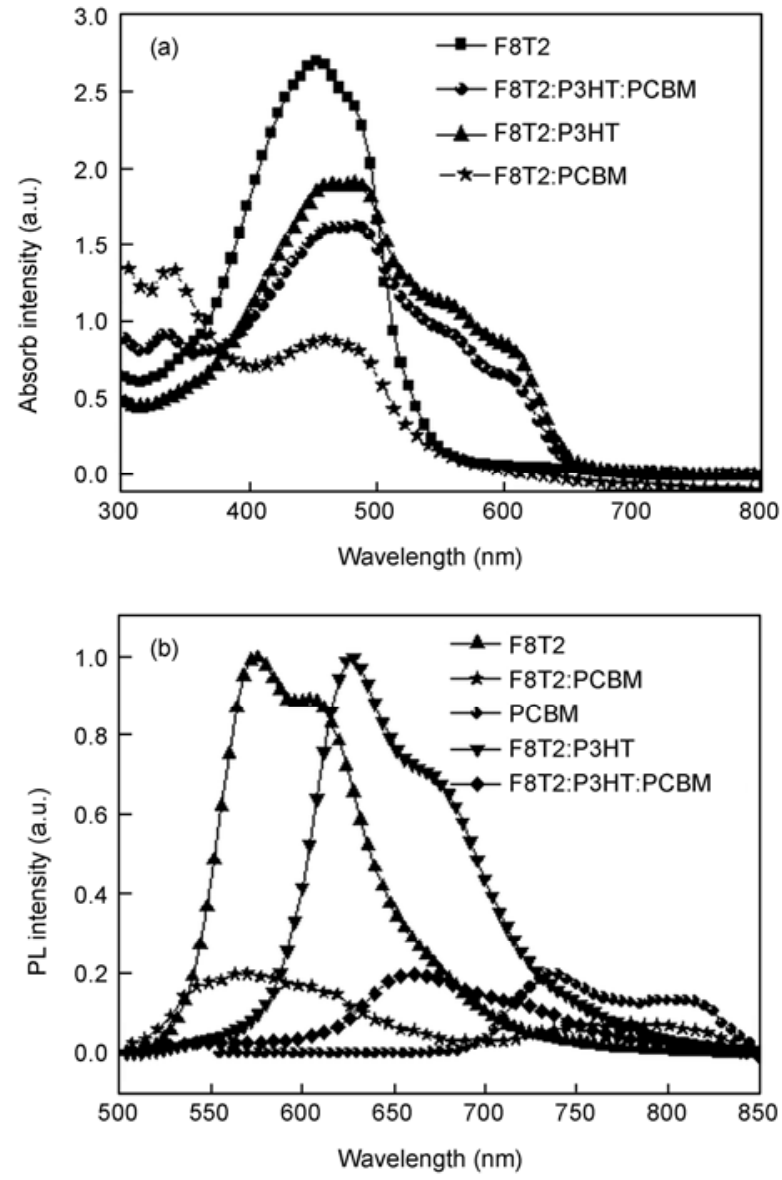

Figure 4 (a) Absorption spectra of F8T2 and F8T2: P3HT before and after doping with PCBM; (b) PL spectra of pure F8T2, F8T2: P3HT before and after doping with PCBM, and PL spectrum of pure PCBM under excitation of $450 \mathrm{~nm}$ light. The PL intensity of all polymers doped with PCBM is magnified more than 100 times for comparison.

The PL spectrum of F8T2 shows one stronger emission peak at $570 \mathrm{~nm}$ and a shoulder emission peak at $605 \mathrm{~nm}$ under excitation by $450 \mathrm{~nm}$ light. The PCBM films show a much weaker emission, which is mainly attributed to a less efficient emission resulting from symmetry-forbidden singlet radiative recombination as well as efficient intersystem crossing to the triplet state [27]. The PL intensity of F8T2 was extensively quenched by doping with PCBM, which is attributed to effective exciton dissociation at the interfaces between F8T2 and PCBM. A similar phenomenon was observed from the co-polymer F8T2: P3HT doped with PCBM.

The $J-V$ characteristics of PSCs based on F8T2:PCBM $(1: 4)$ with different thicknesses of Bphen under white light illumination of $100 \mathrm{~mW} / \mathrm{cm}^{2}$ were measured and are shown in the Figure 5. The $V_{\mathrm{oc}}$ remains about $0.9 \mathrm{~V}$ for most of the fabricated cells with different Bphen thicknesses, and the maximum of $V_{\mathrm{oc}}$ reaches $0.92 \mathrm{~V}$ when the thickness of Bphen is $0 \mathrm{~nm}$. The large $V_{\text {oc }}$ could be supported by the relatively low HOMO levels compared with P3HT, as shown in Figure 1. The $J_{\mathrm{sc}}$ of cells with different Bphen thicknesses

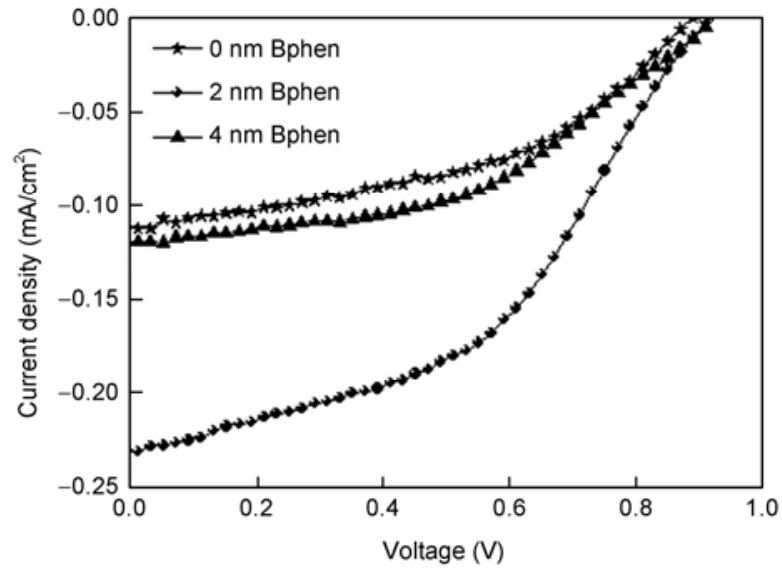

Figure $5 J-V$ curves of cells using F8T2: PCBM as the active layer with different Bphen interlayer thicknesses.

Table 1 Photovoltaic parameters of cells with different Bphen thickness

\begin{tabular}{cccccc}
\hline No. & Bphen $(\mathrm{nm})$ & $V_{\text {oc }}(\mathrm{V})$ & $J_{\text {sc }}\left(\mathrm{mA} / \mathrm{cm}^{2}\right)$ & $\mathrm{FF}(\%)$ & PCE $(\%)$ \\
\hline 1 & 0 & 0.92 & 0.12 & 45.6 & 0.05 \\
2 & 2 & 0.91 & 0.23 & 45.5 & 0.10 \\
3 & 4 & 0.88 & 0.11 & 45.8 & 0.04 \\
\hline
\end{tabular}

is relatively small, which is attributed to its large bandgap $(2.4 \mathrm{eV})$. Hoope et al. pointed out that only $30 \%$ of the photons in the whole solar spectrum have energy higher than $1.9 \mathrm{eV}$ [27]. Therefore, F8T2 absorbs only a small fraction of irradiant light. It is very interesting that the $J_{\text {sc }}$ can be markedly increased from 0.12 to $0.23 \mathrm{~mA} / \mathrm{cm}^{2}$ and then decreased to $0.11 \mathrm{~mA} / \mathrm{cm}^{2}$ when the thickness of the Bphen layer is increased from 0 to $4 \mathrm{~nm}$ in steps of $2 \mathrm{~nm}$. It means that interface engineering can passivate interfacial charge trap states, forbid excitons quenched by metal electrodes, control energy level alignment or enhance charge carrier extraction to optimize $J_{\text {sc }}$, resulting in high efficiency OPV cells [28]. In this experiment, the function of the ultrathin Bphen layer may be forbidding F8T2 excitons quenched by the $\mathrm{Al}$ electrode. However, the $J_{\mathrm{sc}}$ was also decreased with the increase of Bphen thickness due to the larger electron extracting buffer.

Because of the limit of the absorption range of F8T2, another polymer, P3HT, was doped into F8T2:PCBM with a weight ratio of $1: 1: 5$. The same configuration cells were fabricated under the same conditions. The $J-V$ characteristics of the cells were measured and are shown in Figure 6. The main PSC parameters are summarized in Table 2. The $V_{\text {oc }}$ of cells with a co-polymer as the electron donor stayed in the range from 0.8 to $0.85 \mathrm{~V}$, which was decreased by doping with P3HT and was larger than that of P3HT: PCBM (0.6-0.67 V) [27]. The underlying reasons may be: (1) the connection based on F8T2:PCBM and P3HT: 


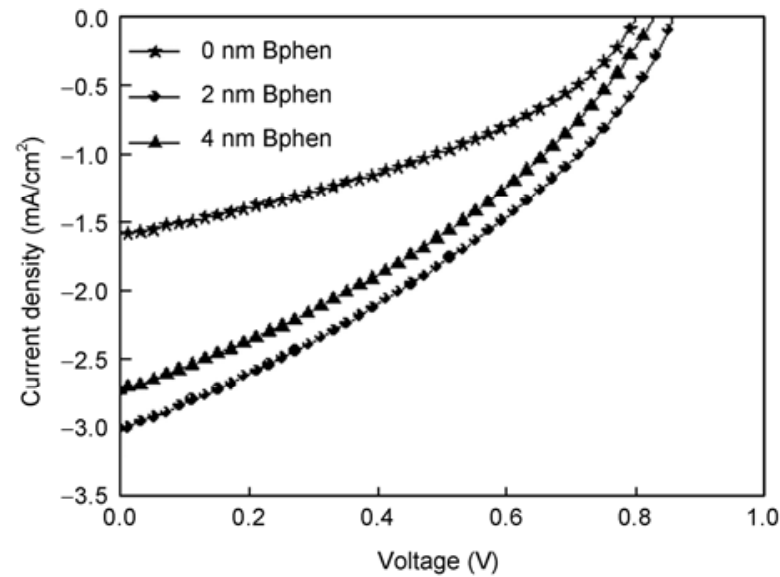

Figure $6 J-V$ curves of cells using F8T2:P3HT:PCBM as the active layer with different Bphen interlayer thicknesses.

Table 2 Photovoltaic parameters of cells with different Bphen thickness

\begin{tabular}{cccccc}
\hline No. & Bphen $(\mathrm{nm})$ & $V_{\mathrm{oc}}(\mathrm{V})$ & $J_{\mathrm{sc}}\left(\mathrm{mA} / \mathrm{cm}^{2}\right)$ & $\mathrm{FF}(\%)$ & PCE $(\%)$ \\
\hline 1 & 0 & 0.82 & 2.73 & 35.3 & 0.79 \\
2 & 2 & 0.85 & 3.02 & 35 & 0.90 \\
3 & 4 & 0.8 & 1.59 & 38.5 & 0.49 \\
\hline
\end{tabular}

PCBM sub-cells, and (2) the energy transfer between F8T2 and P3HT, which is supported by the overlap between the emission spectra of F8T2 and absorption spectra of P3HT as shown in Figure 4. The $J_{\text {sc }}$ of cells with a co-polymer as the electron donor was markedly increased to $3.02 \mathrm{~mA} / \mathrm{cm}^{2}$ and also shows a very similar dependence on the Bphen layer thickness. The marked increase of $J_{\text {sc }}$ is favored by the broader absorption range of $\mathrm{P} 3 \mathrm{HT}$ : F8T2, especially in the longer-wavelength range.

\section{Conclusion}

The luminescent property of F8T2 was studied based on ITO/ PEDOT : PSS/F8T2/Bphen/LiF(0 or $1 \mathrm{~nm}) / \mathrm{Al}$. Both kinds of OLEDs show bright orange emission peaking at $570 \mathrm{~nm}$ and shoulder emission peaking at $605 \mathrm{~nm}$. The EL intensity of OLEDs with $1 \mathrm{~nm} \mathrm{LiF}$ interlayer reaches $45 \mu \mathrm{W}$ under $15 \mathrm{~V}$. The PSCs with F8T2: PCBM as the active layer show a low PCE of $0.10 \%$ with a high $V_{\text {oc }}$ of $0.91 \mathrm{~V}$ and $J_{\text {sc }}$ of 0.23 $\mathrm{mA} / \mathrm{cm}^{2}$. The PSCs using F8T2:P3HT:PCBM as the active layer show a relatively larger $J_{\mathrm{sc}}$ and PCE, which is attributed to the enhanced photon harvesting by P3HT molecules. The ultrathin interfacial Bphen layer plays an important role in improving PSCs performance.

This work was supported by the National Natural Science Foundation of China (10804006 and 21074055) and the Basic Research Foundation of the Central Universities (2011JBM123). F. Zhang is grateful for support from the "Double Hundred Talents Plan" of Beijing Jiaotong University.

1 Chen H Y, Hou J H, Zhang S Q, et al. Polymer solar cells with enhanced open-circuit voltage and efficiency. Nat Photon, 2009, 3: 649-653

2 Liang Y Y, Xu Z, Xia J B, et al. For the bright future-bulk heterojunction polymer solar cells with power conversion efficiency of 7.4\%. Adv Mater, 2010, 22: 1-4

3 Park S H, Roy A, Beaupre S, et al. Bulk heterojunction solar cells with internal quantum efficiency approaching 100\%. Nat Photon, 2009, 3: 297-302

4 Tang W, Hai J, Dai Y, et al. Recent development of conjugated oligomers for high efficiency bulk-heterojunction solar cells. Sol Energy Mater Sol Cells, 2010, 94: 1963-1979

5 Chen L M, Hong Z R, Li G, et al. Recent progress in polymer solar cells: Manipulation of polymer:fullerene morphology and the formation of efficient inverted polymer solar cells. Adv Mater, 2009, 21: 1434-1449

6 Singh T B, Marjanović N, Matt G J, et al. High-mobility n-channel organic field-effect transistors based on epitaxially grown $\mathrm{C}_{60}$ films. Org Electron, 2005, 6: 105-110

7 Zhang F J, Xu X W, Tang W H, et al. Recent development of the inverted configuration organic solar cells. Sol Energy Mater Sol Cells, 2011, 95: 1785-1799

8 Li Y F, Zou Y P. Conjugated polymer photovoltaic materials with broad absorption band and high charge carrier mobility. Adv Mater, 2008, 20: 2952-2958

9 Scharber M C, Wuhlbacher D, Koppe M, et al. Design rules for donors in bulk-heterojunction solar cells-towards $10 \%$ energy conversion efficiency. Adv Mater, 2006, 18: 789-794

10 Dennler G, Scharber M C, Ameri T, et al. Design rules for donors in bulk-heterojunction tandem solar cells-towards $15 \%$ energy conversion efficiency. Adv Mater, 2008, 20: 579-583

11 Bakulin A A, Martyanov D, Paraschuk D Y, et al. Charge-transfer complexes of conjugated polymers as intermediates in charge photogeneration for organic photovoltaics. Chem Phys Lett, 2009, 482: 99-104

12 Lehnhardt M, Riedl T, Rabe T, et al. Room temperature lifetime of triplet excitons in fluorescent host/guest systems. Org Electron, 2011, 12: 486-491

13 Okua T, Nagaoka S, Suzuki A, et al. Formation and characterization of polymer/fullerene bulk heterojunction solar cells. J Phys Chem Solid, 2008, 69: 1276-1279

14 Gowrishankar V, Scully S R, Chan A T, et al. Exciton harvesting, charge transfer, and charge carrier transport in amouphous silicon nanopillar/polymer hybrid solar cells. J Appl Phys, 2008, 103: 064511

15 Norrman K, Madsen M V, Gevorgyan S A, et al. Degradation patterns in water and oxygen of an inverted polymer solar cell. J Am Chem Soc, 2010, 132: 16883-16892

16 Zhang F J, Zhao D W, Zhuo Z L, et al. Inverted small molecule organic solar cells with $\mathrm{Ca}$ modified ITO as cathode and $\mathrm{MoO}_{3}$ modifiedAg as anode. Sol Energy Mater Sol Cells, 2010, 94: 2416-2421

17 Bin J K, Hong J I. Efficient blue organic light-emitting diode using anthracene-derived emitters based on polycyclic aromatic hydrocarbons. Org Electron, 2011, 12: 802-808

18 Zhao D W, Liu P, Sun X W, et al. An inverted organic solar cell with an ultrathin $\mathrm{Ca}$ electron-transporting layer and $\mathrm{MoO}_{3}$ holetransporting layer. Appl Phys Lett, 2009, 95: 153304

19 Zhang F J, Sun F Y, Shi Y Z, et al. Effect of an ultra-thin molybdenum trioxide layer and illumination intensity on the performance of organic photovoltaic devices. Energ Fuel, 2010, 24: 3739-3742

20 Jönsson S K M, Salaneck W R, Fahlman M. Photoemission of $\mathrm{Alq}_{3}$ and $\mathrm{C}_{60}$ films on $\mathrm{Al}$ and LiF/Al substrates. J Appl Phys, 2005, 98: 014901

21 Mason M G, Tang C W, Hung L S, et al. Interfacial chemistry of $\mathrm{Alq}_{3}$ and LiF with reactive metals. J Appl Phys, 2001, 98: 2756

22 Hung L S, Tang C W, Mason M G. Enhanced electron injection in 
organic electroluminescence devices using an $\mathrm{Al} / \mathrm{LiF}$ electrode. Appl Phys Lett, 1997, 70: 152

23 Grozea D, Turak A, Feng X D, et al. Chemical structure of AL/LiF/ Alq interfaces in organic light-emitting diodes. Appl Phys Lett, 2002, 81: 3173

24 Zhang F J, Vollmer A, Zhang J, et al. Energy level alignment and morphology of interfaces between molecular and polymeric organic semiconductors. Org Electron, 2007, 8: 606-614

25 Zhao D W, Tang W H, Ke L, et al. Efficient bulk heterojunction solar cells with poly[2,7-(9,9-dihexylfluorene)-alt-bithiophene] and 6,6- phenyl C61 butyric acid methyl ester blends and their application in tandem cells. ACS Appl Mater Interfaces, 2010, 2: 829-837

26 Zhuo Z L, Zhang F J, Xu X W, et al. Photovoltaic performance improvement of P3HT:PCBM polymer solar cells by annealing treatment. Acta Phys Chim Sin, 2011, 27: 875-880

27 Hoope H, Niggemann M, Winder C, et al. Nanoscale morphology of conjugated polymer/fullerence based bulk heterojunction solar cells. Adv Funct Mater, 2004, 14: 1005-1011

28 Ma H, Yip H L, Huang F, et al. Interface engineering for organic electronics. Adv Funct Mater, 2010, 20: 1371-1388

Open Access This article is distributed under the terms of the Creative Commons Attribution License which permits any use, distribution, and reproduction in any medium, provided the original author(s) and source are credited. 